\title{
Research on Optimization Algorithm of RSSI Positioning Parameters Based on Improved Particle Swarm Optimization
}

\author{
Qiang Ji, Shifeng Zhang and Haoguang Zhao \\ College of Aerospace Science and Engineering, National University of Defense Technology
}

Keywords: Particle swarm optimization algorithm; RSSI; WSN.

\begin{abstract}
The paper put forward to an algorithm based on hybrid mutation particle optimization swarm strategy (HMPOA), it can solve the position coordinates of the unknown nodes. The algorithm uses static sampling to determine the performance index values of particles, then the arc grouping method is used to divide the particle swarm into several subgroups. Finally, the hybrid mutation strategy is used to improve the convergence speed and positioning accuracy of the algorithm, which can overcome the location accuracy of unknown node that overly dependent on the RSSI physical measurement value. Numerical experiments show that the algorithm has fast convergence speed and high positioning accuracy for unknown nodes, and it is feasible for RSSI positioning.
\end{abstract}

\section{Introduction}

Wireless sensor network (WSN) is a network composed of a large number of wireless sensor nodes with cheap, small volume, low energy and communication capabilities. It has been widely used in the fields of national defense, environmental monitoring, target tracking and positioning, production safety and other fields ${ }^{[1]}$. Node positioning technology is one of the basic and key technologies of wireless sensor network applications. The main work of wireless sensor networks is to perform various monitoring tasks in the designated area. Location information is very important for sensor network monitoring. Location information is an important information contained in sensor node monitoring messages. The accuracy of location information directly affects the effectiveness of sensor nodes' data acquisition ${ }^{[2]}$. Therefore, it is meaningless to obtain data from node monitoring without knowing the location information of the node.

At present, localization algorithms in wireless sensor networks are divided into Range-based) localization algorithm and Range-free localization algorithm ${ }^{[3-5]}$. The positioning precision based on distance is unsuitable for large-scale network than higher precision location with extra hardware facilities and high cost. The precision is relatively low without the location algorithm, but it does not need the location with low energy consumption, low communication overhead, no hardware support and low cost and so on. Free positioning is more suitable for largescale network and future development.
However, because the RSSI value is greatly influenced by the surrounding environment, the collected signal values often have large errors, so it needs to be noise treatment for unknown nodes in first phase. Recently, the research of node location algorithm based on RSSI has been widely concerned, and many new efficient algorithms have emerged, including distance correction, curve fitting and intelligent optimization algorithm ${ }^{[2-5]}$. The distance correction method is a distance error estimation model, which can be used to modify the distance measurement value according to the distribution characteristics of measured noise. Ren Weizheng and other ${ }^{[1]}$ proposed a RSSI Distance Difference Localization Algorithm (DDLA), which takes the nearest anchor node from the unknown node as a reference node, and makes a differential correction in the RSSI range. The algorithm can effectively eliminate the measurement noise of RSSI, but the location accuracy of unknown nodes needs to be improved. Curve fitting is a method of data fitting through selecting the appropriate model structure. Yu Jimin et al. ${ }^{[2]}$ uses the scalar Calman filter to suppress the noise of original RSSI data, striving to make the RSSI value matched well with the distance length value; Zhang Bin ${ }^{\text {[3- }}$ ${ }^{5]}$ preprocess the original RSSI data by filtering technology, and using adaptive piecewise curve fitting to obtain the environmental parameters and establish the signal attenuation under different environments. The least square method is used to calculate the nodes coordinates, Although the location accuracy is high, the environmental parameters are difficult to adapt to the changes of complex 
environment, this makes the estimation of node position coordinates more serious.

Aiming at the node location problem of wireless sensor networks, this paper establishes a random optimization model with noise interference, and proposes a Hybrid Mutation-based particle swarm Optimization Algorithm (HMPOA) to solve the location coordinates of the unknown nodes, the algorithm uses the static sampling to determine the performance index of the particle, uses the arc grouping method to divide the particle swarm into several subgroups, and uses the mixed mutation strategy to improve the convergence speed and positioning accuracy, and can overcome the problem that the location accuracy of the unknown node is overly dependent on the RSSI physical measurement value.

\section{Description of RSSI location model}

RSSI positioning is a signal intensity indicator $(\mathrm{dBm})$ to estimate the coordinates of unknown nodes, which adopt signal receiving terminal receives RSSI transmit by signal transmitting terminal. The distance relationship $d(m)$ between RSSI and unknown node is usually described by logarithmic loss function. That is:

$$
R S S I=A-10 k \log d+\xi^{n}, \xi \sim N\left(0, \sigma^{2}\right)
$$

In the formula, $A$ is the signal intensity received when the signal is transmitted by the unit. $K$ is the path loss index, and its value is related to the environment. $\xi$ indicates the degree of environment influence on the theoretical RSSI value. $\mathrm{n}$ represents the number of interfering signal sources. The coordinates of unknown node $\mathrm{P}$ are $(x, y)$, The node coordinates of node $i(i=1,2, \cdots, m)$ are $\left(x_{i}, y_{i}\right)$, and the real distance between node $\mathrm{P}$ and anchor node $i$ is:

$$
d_{i}=\sqrt{\left(x-x_{i}\right)^{2}+\left(y-y_{i}\right)^{2}}
$$

In combination (1), the estimated value $D_{i}$ is:

$$
\log \hat{d}_{i}=\left(A-R S S I_{i}+\xi_{i}^{n}\right) / 10 k, \mathrm{i}=0 \sim \mathrm{n}
$$

Among them, RSSIi is the wireless signal intensity that unknown node receives the signal transmitted by anchor node i. $\xi_{i}$ is a random variable with the same $\xi$ distribution as above. Thus, the average distance error function between nodes $\mathrm{P}$ to $\mathrm{m}$ anchor nodes is:

$$
\begin{gathered}
e\left(x, y, \xi^{n}\right)=\frac{1}{m} \sum_{i}^{m} \mid \sqrt{\left(x-x_{i}\right)^{2}+\left(y-y_{i}\right)^{2}}- \\
10^{\left(A-R S S I_{i}+\xi^{n}\right) / 10 k} \mid
\end{gathered}
$$

$\xi=(\xi 1, \xi 2, \ldots, \xi m)$. Therefore, the RSSI localization problem of unknown nodes can be transformed into the following two-dimensional expectation value problem (EVP):

$$
\min \left(E_{i}\left[f\left(x, \xi^{n}\right)\right]\right), i=0 \sim n
$$

$x$ is a $\mathrm{p}$ dimension decision vector in a $R p$ space. $\xi$ is $\mathrm{m}$ dimensional random vector. When the sample $\xi$ size is designated as $\mathrm{M}$, the expected target value of candidate solution $\mathrm{x}$ is estimated from the bottom.

$$
\hat{f(x)}=\frac{1}{M} \sum_{n=1}^{M} f\left(x, \xi^{n}\right)
$$

Among them, $\xi j$ is $j$ observation vector value of random $\xi$ vector.

\section{New particle optimization algorithm}

The main idea of the algorithm is to estimate performance index of particles by static sampling, and to divide the particle population into three seed groups by using ascending power arrangement and arc grouping of particles, and then use the mixed mutation strategy to update the subgroups.

The description of algorithm HMPOA is as follows:

Step. 1 Setting parameters: population size N, sample capacity $\mathrm{M}$, maximum iterated algebra Gmax, fixed step length $\theta$, adaptive factor $\eta$.

Step. 2 Set $\mathrm{t} \leftarrow 1$, the initial population $\mathrm{P}$ was randomly generated with a scale of $\mathrm{N}$.

Step. 3 According to the sample size M and formula (4), we calculate the target estimation value in population $\mathrm{P}$ particles, and note the particle with minimum estimated value in particles $\mathrm{P}$ is xbst and the maximum estimated value is xwst.

Step.4 According to the estimated value of the target, the particles in the $\mathrm{P}$ are descended to power, and the first $13 \mathrm{~N}$ particles are assigned to the high quality population $\mathrm{A}$. The remaining particles form the population $\mathrm{B}$ and $\mathrm{C}$ according to the above arc grouping method.

Step.5.1 Population A, B, C update:

$$
x_{j}^{\prime} \leftarrow x_{b s t, j}+\lambda \theta\left(1-\frac{t}{G_{\max }+1}\right)^{\eta},
$$

$$
\left\{\begin{array}{c}
x^{\prime}, \hat{f}\left(x^{\prime}\right)<\hat{f}(x) \\
x_{b s t}+\xi, \hat{f}\left(x^{\prime}\right) \geq \hat{f}(x)
\end{array}\right.
$$

Wherein: $j=1,2, \ldots, p$ and $x^{\prime}=\left(x_{1}^{\prime}, x_{2}^{\prime}, \ldots, x_{p}^{\prime}\right)$, $\xi$ is $\mathrm{P}$ dimensional random vector in $[-1,1], \lambda$ is random variable in $[-1,1], \hat{f}\left(x^{\prime}\right)$ is target estimation value for particle $x^{\prime}$ in sample size $M$.

Step.5.2 In population $\mathrm{B}$, each particle $\mathrm{x}$ is updated to $\mathrm{y}$ according to the pattern of homogeneous variation, and the population $\mathrm{B}^{*}$ is obtained:

$$
\begin{gathered}
x_{j}^{\prime}=\alpha x_{j}+(1-\alpha) x_{A, j}+\lambda \theta\left(1-\alpha^{1-t / T+1}\right) \\
y=\left\{\begin{array}{c}
x^{\prime}, \alpha<0.5 \\
x+I \lambda \theta\left(1-\alpha^{1-t / T+1}\right), \alpha \geq 0.5
\end{array}\right.
\end{gathered}
$$

Wherein: $x_{A, j}$ is the jth component of the particle $x_{A}$ in the population in $\mathrm{A}, \alpha$ and $\lambda$ is random variable respectively in $[0,1]$ and $[-1,1]$, I represents the $p$ dimensional vector with all elements 1 . 
Step.5.3 each particle $\mathrm{x}$ in population $\mathrm{C}$ is updated as follows to obtain the population $\mathrm{C}^{*}$ :

$$
\mathrm{x}_{j}^{\prime}=\left\{\begin{array}{c}
x_{j}+\left((2 \alpha)^{t / 2 G_{\max }}-1\right)\left(x_{b s t}-x_{j}\right), \alpha<0.5 \\
x_{j}+\left(1-(2(1-\alpha))^{t / 2 G_{\max }}\right)\left(x_{j}-x_{w s t, j}\right), \alpha \geq 0.5
\end{array}\right.
$$

Wherein: $a$ is random value in $[0,1]$, the calculation of $f\left(x^{\prime}\right)$ is the same as above.

Step.6 Executive group update: $\mathrm{P} \leftarrow \mathrm{A}^{*} \cup \mathrm{B}^{*} \cup \mathrm{C}^{*}$; according to the sample size $\mathrm{M}$, we calculate the target estimate of the particle in $\mathrm{P}$, and update $\mathrm{x}_{\mathrm{bst}}$ and $\mathrm{x}_{\mathrm{wst}}$.

Step.7 Set $\mathrm{t} \leftarrow \mathrm{t}+1$; if $\mathrm{t}<\mathrm{Gmax}$, then goto step.4; otherwise, output $\mathrm{x}_{\mathrm{bst}}$ 。

\section{Simulation results and analysis}

100 anchor nodes are randomly distributed in a $100 \mathrm{~m} \mathrm{x}$ $100 \mathrm{~m}$ rectangular area, and the coordinates of the unknown nodes are set to $(50 \mathrm{~m}, 60 \mathrm{~m})$, and all the anchor nodes are within the communication range of the unknown nodes. It is assumed that the mean value of the RSSI measurement is 0 and the Gauss noise with a mean square deviation $\sigma$. We select $\sigma=0.2,0.4, \ldots, 1$. Under the different values $\sigma$, each algorithm runs 100 times independently. The relation between the mean errors and sigma obtained by each algorithm is shown in Figure 1. The results obtained are shown in Table 2 (only with sigma $=0.2$ and 0.8 as example).

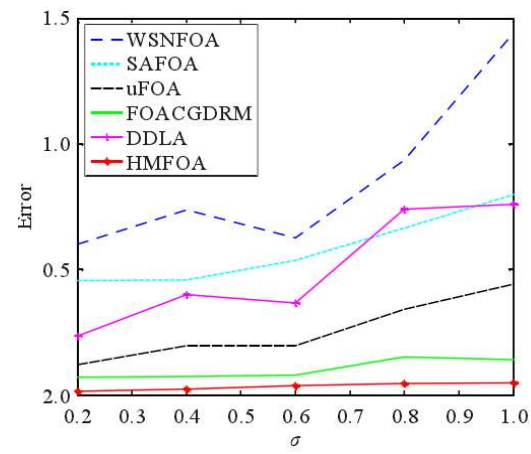

Figure 1. The relation between the Error mean and $\sigma$.

From Figure 2, we can see that the average Error obtained by the above algorithm is directly proportional to the mean variance $\sigma$, and the noise has a greater impact on the search algorithm quality. When the mean square error of noise is large, the deviation between the measured value of RSSI and the true RSSI value is large, and the solution quality obtained by the algorithm is poor. By comparison, noise has very small interference to HMFOA, so the location estimation for unknown nodes is more accurate; DDLA is greatly disturbed by noise, so that the error is larger, the positioning precision is low, and the WSNFOA is disturbed by the noise most.

In order to detect the coordinate estimation effect of HMFOA on the unknown nodes in the actual environment of RSSI, the algorithm runs 100 times independently, and the results obtained are shown in Table 1 . In the experiment, the coordinates of the anchor nodes are $(0,0)$, $(5.4,0),(0,5.4),(6,3.6)$, and the unit is $m$, and the actual coordinates of unknown nodes are $(2.4,3.6)$.

Table 1. Statistical results in actual environment.

\begin{tabular}{|c|c|c|c|c|c|c|c|}
\hline \multirow{3}{*}{ Algorithm } & \multicolumn{2}{|c|}{ Solution $(X, Y)$} & \multicolumn{4}{|c|}{ Error } & \multirow{2}{*}{$A T / \mathrm{s}$} \\
\hline & Worst & Best & $\operatorname{Max}$ & $\operatorname{Min}$ & Mean & St.Dev & \\
\hline & \multicolumn{7}{|c|}{ Empty classroom } \\
\hline WSNFOA & $(3.09,3.04)$ & $(2.41,3.59)$ & 0.8866 & 0.0198 & 0.0853 & 0.0342 & 3.17 \\
\hline SAFOA & $(4.54,13.00)$ & $(2.41,3.60)$ & 3.1459 & 0.0120 & 0.1916 & 0.2955 & 3.15 \\
\hline$\mu \mathrm{FOA}$ & $(2.32,3.46)$ & $(2.42,3.59)$ & 0.1580 & 0.0200 & 0.1056 & 0.0022 & 1.39 \\
\hline FOACGDRM & $(2.32,3.47)$ & $(2.41,3.59)$ & 0.1275 & 0.0204 & 0.0404 & $5.09 \mathrm{E}-04$ & 3.15 \\
\hline HMFOA & $(2.38,3.54)$ & $(2.42,3.59)$ & 0.0660 & 0.0200 & 0.0387 & $7.11 \mathrm{E}-05$ & 1.68 \\
\hline
\end{tabular}

It can be seen from table 1 that the positioning precision of HMFOA is the highest in the empty classroom environment, FOACGDRM times, and the positioning precision of SAFOA is the worst. In addition, the error comparison obtained from table 3 shows that compared with HMFOA, the algorithm stability is very good and can better apply HMFOA to the RSSI positioning problem.

\section{Conclusion}

In order to solve the precision problem of RSSI physical measurement, the location problem of unknown nodes of the wireless sensor is described as an unconstrained expected value programming problem. The algorithm structure is simple and easy to apply. The search efficiency depends on the sample size, population size and dimension of random variables. Comparative experimental results show that the algorithm has high positioning accuracy, good convergence and fast search speed for unknown nodes. The actual scenario test shows that the algorithm is feasible for solving the RSSI location problem. In the future, we will further optimize the structure of the algorithm, improve its execution efficiency, and strive to meet the needs in actual environment.

\section{References}

1. Ren Weizheng, Xu Lianming, Deng Zhongliang, and so on. Range difference correction location algorithm based on RSSI []]. Journal of sensing technology, 2008, 21 (7): 1247-1250. (In Chinese)

2. Yu Jimin, Wang Haiyun, Tang Xianlun. WSNs point positioning method for improved particle optimization algorithm []]. Microelectronics \&computers, 2014 (11): 111-115. (In Chinese) 
3. Zhang Bin, Zhang Damin, Amin Han. Particle Optimization Algorithm Based on simulated annealing [J]. Computer applications, 2016, 36 (11): 3118-3122. (In Chinese)

4. Zhang Xiaolu, Zhang Zhuhong. Particle swarm optimization algorithm for multimodal function optimization [J]. Information and control, 2016 (3): 361-370. (In Chinese)

5. Du Xiaoxin, Zhang Jianfei, Guo Yuan, etc. Particle Optimization Algorithm Based on Cauchy Gauss dynamic subtraction mutation [J]. Computer engineering and science, 2016, 38 (6): 11711176. (In Chinese) 